\title{
Global Symmetry Restoration in High Temperature Higgs Theories
}

 \\ Joseph Henry Laboratories, Department of Physics, Princeton University, Princeton, NJ 08544, \\ USA
}

\begin{abstract}
A simple high temperature expansion is developed for lattice gauge theories with scalar matter fields. The expansion is used to prove the absence of global symmetry breaking for sufficiently high temperature.
\end{abstract}

\section{Introduction}

In both statistical mechanics and quantum field theory there are many examples of theories with global symmetries which are spontaneously broken at zero temperature. Typically, one expects such symmetries to be restored at sufficiently high temperature. The purpose of this paper is to prove the absence of spontaneous symmetry breaking at high temperature in a wide class of theories which includes gauge theories with bosonic matter fields (commonly called Higgs theories).

In the case of abelian Higgs theories, previous work by Kennedy and King [7] proves the existence of a low temperature phase with spontaneously broken global gauge invariance. Combined with our result, this implies that a phase transition must separate the unbroken symmetry high temperature phase from the broken symmetry low temperature phase. To our knowledge, this provides the first rigorous demonstration that the Higgs phenomenon in abelian gauge theories is associated with a genuine finite temperature phase transition.

Our proof of the absence of high temperature symmetry breaking is also applicable to non-abelian Higgs theories, however in such theories there is no corresponding demonstration of the breaking of global gauge symmetry at low temperature. (The abelian proof cannot be generalized to non-abelian theories for several reasons. These include the fact that the order parameter used in abelian theories - essentially equal to the Higgs field in Landau gauge - is not well defined in non-abelian theories. In addition, in some non-abelian theories there are strong

* Research supported in part by U.S. National Science Foundation grant PHY 8117463

$\star \star$ Research supported in part by the Department of Energy under grant No. DE-AC0276ER 03072

$\star \star \star$ Alfred P. Sloan Research Fellow 
arguments suggesting that the low and high temperature phases are continuously connected $[4,1]$.)

We will use a regularized Euclidean functional integral with periodic boundary conditions in the (imaginary) time direction to represent a finite temperature field theory. This framework has been previously used to prove, for example, the absence of confinement in high temperature pure gauge theories $[2,10]$. Our approach will be similar to that used in the proof that chiral symmetry cannot be spontaneously broken in $S U$ (2) gauge theories with dynamical massless fermions at high temperature [10]. Specifically, we will introduce a cluster expansion for the scalar fields and show that this expansion is convergent for sufficiently large temperature. This expansion will imply that the correlations of the scalar field are exponentially damped at large distance. Consequently, no order parameter built out of the scalar field can show non-zero magnetization.

Our cluster expansion embodies the simple physical idea that at large temperature thermal fluctuations will create sufficient disorder to overcome any ordering present at zero temperature. In order to prove the convergence of our expansion, we will exploit the positivity of the euclidean functional integral. We will not require the presence of either reflection positivity [5] or correlation inequalities [6].

In Sect. II, we begin by discussing the simple case of pure scalar field theories (or equivalent classical spin models). Then in Sect. III we present the generalization to gauge theories with matter fields. Finally in Sect. IV we apply our results to the case of non-compact abelian Higgs theories.

\section{Symmetry Restoration for Scalar Fields at High Temperature}

Our finite temperature field theory may be represented by a Euclidean functional integral with periodic boundary conditions in the imaginary time direction. We will use a lattice regularization to define the functional integral.

Let $\Lambda$ be the $(d+1)$-dimensional lattice on which the scalar field theory is defined. The lattice $\Lambda$ will be anisotropic, with spacing $a$ in the time direction and unit spacing in the $d$ spatial directions. This choice is made because all our estimates will be uniform in $a$, and so we will take the time continuum limit at the end. Unfortunately there is no easy way to let the remaining spatial lattice spacings approach zero, so these spacings are fixed to be one (any other non-zero spacing can be reached by rescaling the parameters of the theory). We will choose our boundary conditions so that the lattice is periodic in the time direction. Our results are independent of the boundary conditions in the spatial directions, and of the lattice length in any spatial direction. The lattice length in the time direction is $\beta$, the inverse temperature $T^{-1}$. Hence, the number of sites in the time direction, $N_{t}$, equals $\beta / a$. Note that the physical temperature $T=\left(N_{t} a\right)^{-1}$ may be varied continuously by changing the timelike lattice spacing $a$ (for fixed $N_{t}$ ), or discretely by changing $N_{t}$. We will generally express our answers in terms of the inverse temperature $\beta$ and the lattice spacing $a$; however it should be remembered that the ratio $\beta / a=N_{t}$ must be a positive integer. In the time continuum limit the timelike lattice spacing $a$ is sent to zero, and the number of sites $N_{t}$ to infinity, in such a way that the physical temperature $T$ remains fixed. Our results concerning symmetry 
restoration will be valid for sufficiently small inverse temperature $\beta$ independent of the lattice spacing $a$ (or equivalently $N_{t}$ ).

Let $\phi$ be a real (or complex) $N$-component scalar field on the lattice $\Lambda$. The theory will be $O(N)$ [or $U(N)$ ] invariant, and we will assume a double-well potential for $\phi$. This means that at zero temperature we can expect symmetry breaking. Specifically the lattice action is ${ }^{1}$

$$
S(\phi)=\frac{1}{2} \sum_{b \in \Lambda^{*}} a|\partial \phi(b)|^{2}-\sigma \sum_{x \in \Lambda} a|\phi(x)|^{2}+\lambda \sum_{x \in \Lambda} a\left(|\phi(x)|^{2}\right)^{2},
$$

where $|\phi|^{2}=\sum_{i=1}^{N}\left|\phi_{i}\right|^{2}$ and $\lambda, \sigma$ are positive constants. We have denoted by $\Lambda^{*}$ the set of bonds $b$ on the lattice. For each spacelike bond $b=\langle x, y\rangle$ the derivative is

$$
\partial \phi(b)=\phi(y)-\phi(x),
$$

while for a timelike bond $b^{\prime}=\left\langle x^{\prime}, y^{\prime}\right\rangle$,

$$
\partial \phi\left(b^{\prime}\right)=a^{-1}\left(\phi\left(y^{\prime}\right)-\phi\left(x^{\prime}\right)\right) .
$$

The expectation of any observable $F(\phi)$ is defined as

$$
\langle F\rangle=Z^{-1} \int \prod_{x \in A} d \phi(x) \exp [-S(\phi)] F(\phi),
$$

where $Z$ is chosen to normalize the measure. This expectation depends on the inverse temperature $\beta$ through our choice of boundary conditions in (2.1). Of course it also depends on the parameters $\lambda, \sigma$ and the lattice spacing $a$.

When $\sigma$ and $\beta$ are both large, the theory given by (2.4) is expected to have symmetry breaking. In particular, let $z$ and $w$ be any two spacelike separated sites on $\Lambda$. Then the two point function $\left.\overline{\langle\phi(z)} \cdot \phi(w)\rangle=\sum_{i=1}^{N} \overline{\left\langle\phi_{i}(z)\right.} \phi_{i}(w)\right\rangle$ can be used to test for symmetry breaking. In the limit where the spatial lattice size becomes infinite, we define

$$
\left.M^{2}=\lim _{|z-w| \rightarrow \infty} \lim _{\beta \rightarrow \infty} \overline{\langle\phi(z)} \cdot \phi(w)\right\rangle .
$$

For $\sigma$ sufficiently large, it has been proven that $M^{2}>0$ for $d \geqq 1$ in the case of a real field $\phi(x)$ with one component [6]. When $\phi(x)$ has more than one real component, or is complex, infrared bounds show that $M^{2}>0$ for $d \geqq 2$, again for $\sigma$ sufficiently large [5]. (These statements hold for $T=0$ and $a<\infty$, including the time continuum limit where $a \rightarrow 0$. If $N_{t}=\beta / a$ is held fixed then $d$ must be replaced by $d-1$ in the previous statements.)

These results show that the global $O(N)$ [or $U(N)$ ] symmetry of the theory given by (2.1) is broken at zero temperature. In this paper we prove that in the theory given by the same action (2.1) at sufficiently high temperature, the full $O(N)$ [or $U(N)]$ symmetry is present. This implies that the theory has a symmetrybreaking phase transition at some intermediate temperature. This result holds for all values of $N$, and more remarkably it is uniform in the temporal lattice spacing $a$.

${ }^{1}$ Generalizing our results to theories with arbitrary $O(N)$ [or $U(N)$ ] invariant polynomial interactions is straightforward 
Therefore it implies the same result for the time continuum limit of the theory. Our result is stated below as Theorem 2.1 and is proved in the remainder of this section.

Theorem 2.1. For any choice of parameters $\lambda, \sigma, a, N$ and any dimensional $d \geqq 1$, there is a temperature $0<T^{*}<\infty$ and a function $M(T)$, both depending only on $\lambda, \sigma, N, d$, such that for all $T>T^{*}$,

$$
\langle\bar{\phi}(z) \cdot \phi(w)\rangle \leqq c N \sqrt{\frac{T}{\lambda}} \exp [-M(T)|z-w|],
$$

where $z, w$ are any spacelike separated lattice sites and where $M(T) \rightarrow \infty$ as $T \rightarrow \infty$ (c is a numerical constant).

Remarks. (1) This result agrees with perturbative calculations of the effect of finite temperature on symmetry breaking in scalar field theories $[3,11]$.

(2) Since our result is independent of the temporal lattice spacing $a$, this parameter may be taken to zero. Theorem 2.1 is then a statement about the finite-temperature behavior of a quantum mechanical system of coupled anharmonic oscillators.

(3) We will see that for Theorem 2.1 to hold, $\beta=1 / T$ must satisfy the bounds

$$
\beta \leqq \min \left\{\frac{\lambda}{\sigma^{2}}, \lambda^{-1 / 3}\right\} \text {, and } \frac{\beta}{\lambda} \text { sufficiently small. }
$$

It should be pointed out that these bounds do not predict the correct dependence of $\beta$ on $\lambda$ when $\lambda$ is large. Since we are not interested in the limit $\lambda=\infty$, we do not pursue this point.

(4) When $\phi(x)$ is a fixed length spin, all our results are considerably easier to prove.

Proof of Theorem 2.1. The idea of the proof is to consider the model as a perturbation of decoupled anharmonic oscillators located at the sites of the spatial lattice. At high temperature those oscillators are almost independent and so the long range order is destroyed.

At this point it is convenient to define a decoupled measure for each oscillator on the spatial lattice. We will denote by $\Omega$ some fixed spatial sublattice of $\Lambda$, that is the set of all sites in $\Lambda$ with a given time coordinate. So $\Omega$ is a $d$-dimensional lattice. Of course each site $x \in \Omega$ is strongly coupled (with periodic boundary conditions) to every other site in $\Lambda$ with the same spatial coordinates. We will write $\Lambda_{x}$ to denote all those sites obtained by translating $x$ in the time direction.

Then for each $x \in \Omega$ we define the measure

$$
\begin{aligned}
d \mu_{x}(\phi)= & z^{-1} \prod_{u \in \Lambda_{x}} d \phi(u) \\
& \times \exp \left[-\frac{1}{2} \sum_{b \in \Lambda_{x}^{*}} a|\partial \phi(b)|^{2}+(\sigma-2 d) \sum_{u \in \Lambda_{x}} a|\phi(u)|^{2}-\lambda \sum_{u \in \Lambda_{x}} a\left(|\phi(u)|^{2}\right)^{2}\right],
\end{aligned}
$$

where once again $\Lambda_{x}^{*}$ denotes the bonds in $\Lambda_{x}$. Also $z^{-1}$ is the obvious normalization for the measure.

The only remaining terms in the action (2.1) are interactions between sites with different spatial coordinates. For each bond $b=\langle x, y\rangle$ in $\Omega^{*}$, we denote by $\Lambda_{b}^{*}$ all 
those bonds obtained from $b$ by translations in the time direction. Combining all such terms for a given $b \in \Omega^{*}$ gives the interaction

$$
V(b)=\frac{1}{2} \sum_{\langle u, v\rangle \in \Lambda_{b}^{*}}|\phi(u)+\phi(v)|^{2} .
$$

Notice that we have chosen the single site measure (2.7) in such a way that (2.8) is positive for all values of $\phi$. This will be very useful when we have to estimate ratios of partition functions.

With these definitions the partition function of the theory can be written as

$$
\begin{aligned}
Z & =\int \prod_{x \in \Omega} d \mu_{x}(\phi) \exp \left[\sum_{b \in \Omega^{*}} V(b)\right] \\
& \equiv \int d \mu(\phi) \exp \left[\sum_{b \in \Omega^{*}} V(b)\right],
\end{aligned}
$$

and the two-point function is

$$
\overline{\langle\phi(z)} \cdot \phi(w)\rangle=Z^{-1} \int d \mu(\phi) \exp \left[\sum_{b \in \Omega^{*}} V(b)\right] \overline{\phi(z)} \cdot \phi(w) .
$$

In this form the model may be analysed using a standard high-temperature expansion [9]. We first define for each $b \in \Omega^{*}$,

$$
1+\varrho(b)=\exp V(b) .
$$

Then (2.10) becomes

$$
\begin{aligned}
Z & =\int d \mu(\phi) \prod_{b \in \Omega^{*}}[1+\varrho(b)], \\
& =\sum_{Y \subset \Omega^{*}} k(Y),
\end{aligned}
$$

where the sum is over all subsets of $\Omega^{*}$ and

$$
k(Y)=\int d \mu(\phi) \prod_{b \in Y} \varrho(b) .
$$

Inserting the same expansion into (2.11) gives

$$
\overline{\langle\phi(z)} \cdot \phi(w)\rangle=Z^{-1} \sum_{Y \subset \Omega^{*}} k^{\prime}(Y)
$$

with the obvious definition of $k^{\prime}(Y)$. Each such subset $Y$ may be written as the disconnected union of two sets $X$ and $W$, where the set $W$ is connected and we choose $w \in W$ ( $W$ may be empty):

$$
Y=X \cup W .
$$

Because $d \mu(\phi)$ factorises over sites in $\Omega$, any term in (2.16) for which $z \notin W$ will vanish [remember the measure (2.7) is even]. So (2.16) becomes

$$
\overline{\langle\phi(z)} \cdot \phi(w)\rangle=Z^{-1} \sum_{\substack{W \subset \Omega^{*} \\ z, w \in W \\ \text { connected }}} k^{\prime}(W) \sum_{X \subset \Omega^{*} \mid \bar{W}} k(X),
$$


where $\bar{W}$ is the "closure" of $W$, i.e., all those bonds in $\Omega^{*}$ which share an endpoint with some bond in $W$. It is clear from (2.14) that we can write

$$
\sum_{X \subset \Omega^{*} \backslash \bar{W}} k(X)=Z\left(\Omega^{*} \backslash \bar{W}\right),
$$

where $Z\left(\Omega^{*} \backslash \bar{W}\right)$ is the partition function of the full model with interactions on bonds in $\bar{W}$ set to zero. Therefore (2.17) becomes

$$
\overline{\langle\phi(z)} \cdot \phi(w)\rangle=\sum_{\substack{W \subset \Omega^{*} \\ z, w \in W \\ \text { connected }}} k^{\prime}(W) \frac{Z\left(\Omega^{*} \backslash \bar{W}\right)}{Z} .
$$

Our proof now reduces to the following two propositions.

Proposition 2.2. For any choice of parameters $\lambda, \sigma, a, N$ and dimension $d \geqq 1$, there is $a$ temperature $0<T_{1}^{*}<\infty$ and a function $M^{\prime}(T)$, both depending only on $\lambda, \sigma, N, d$, such that for all $T>T_{1}^{*}$

$$
\left|k^{\prime}(W)\right| \leqq c^{\prime} N \sqrt{\frac{T}{\lambda}} \exp \left[-M^{\prime}(T)|W|\right],
$$

where $W \subset \Omega^{*}$ is connected and $|W|$ is the number of bonds in $W$. Furthermore $M^{\prime}(T)$ $\rightarrow \infty$ as $T \rightarrow \infty$.

Proposition 2.3. For any values of the parameters of the theory, and for any subset $W \subset \Omega^{*}$,

$$
\frac{Z\left(\Omega^{*} \backslash \bar{W}\right)}{Z} \leqq 1
$$

Before proving these propositions we will use them to complete Theorem 2.1. Let $R=|z-w|$. Then from (2.19), (2.20), and (2.21),

$$
\overline{\mid\langle\phi(z)} \cdot \phi(w)\rangle\left|\leqq \sum_{\substack{W \subset \Omega^{*} \\ z, w \in W \\ \text { connected }}}\right| k^{\prime}(W) \mid \leqq c^{\prime} N \sqrt{\frac{T}{\lambda}} \sum_{n=R}^{\infty} \sum_{\substack{W:|W|=n \\ z \in W \\ \text { connected }}} \exp \left[-M^{\prime}(T) n\right] .
$$

By a well-known argument [8] (useful for escaping from mazes), a connected set $W$ containing $n$ bonds can be traversed by a walk of length $2 n$ which crosses every bond exactly twice. So the sum over connected sets $W$ is bounded by a sum over random walks of length $2 n$ which begin at $z$, and the number of those is at most $(2 d)^{2 n}$. Therefore provided $T$ is sufficiently large so that $M^{\prime}(T)>2 \ln (2 d)$, we have

$$
\overline{\langle\phi(z)} \cdot \phi(w)\rangle \mid \leqq c^{\prime} N \sqrt{\frac{T}{\lambda}} \sum_{n=R}^{\infty}(2 d)^{2 n} \exp \left[-M^{\prime}(T) n\right] \leqq c N \sqrt{\frac{T}{\lambda}} \exp [-M(T) R],
$$

where $M(T)>0$ and $M(T) \rightarrow \infty$ as $M^{\prime}(T) \rightarrow \infty$. This completes Theorem 2.1.

Proof of Proposition 2.2. Recall that

$$
k^{\prime}(W)=\int d \mu(\phi) \prod_{b \in W} \varrho(b) \overline{\phi(z)} \cdot \phi(w) .
$$

From (2.12) we have

$$
|\varrho(b)| \leqq|\exp V(b)-1| \leqq|V(b)| \exp V(b) .
$$


If $b=\langle x, y\rangle$ we get by Schwarz's inequality for (2.8)

$$
V(b) \leqq \sum_{u \in \Lambda_{x}} a|\phi(u)|^{2}+\sum_{v \in \Lambda_{y}} a|\phi(v)|^{2} .
$$

Expanding out the product over bonds in $W$ gives

$$
\prod_{b \in W} V(b) \leqq \sum_{\{q(x)\}} \prod_{x \in \Omega}\left\{\sum_{u \in \Lambda_{x}} a|\phi(u)|^{2}\right\}^{q(x)} .
$$

The sum in (2.25) is over choices of integer $q(x)$ for each $x \in \Omega$, with the constraints that $q(x)=0$ outside $W, 0 \leqq q(x) \leqq 2 d$ for each $x$, and most importantly

$$
\sum_{x \in \Omega} q(x)=|W| .
$$

Also for each $x \in \Omega$ we let $p(x)$ be the number of bonds in $W$ with endpoints at $x$. Then substituting (2.24), (2.25) into (2.22), and using the bound

$$
|\bar{\phi}(z) \cdot \phi(w)| \leqq|\phi(z)|^{2}+|\phi(w)|^{2},
$$

we obtain the expansion

$$
\begin{aligned}
\left|k^{\prime}(W)\right| \leqq & \sum_{\{q(x)\}} \int d \mu(\phi)\left(|\phi(z)|^{2}+|\phi(w)|^{2}\right) \prod_{x \in \Omega} \\
& \times \exp \left[p(x) \sum_{u \in \Lambda_{x}} a|\phi(u)|^{2}\right]\left\{\sum_{u \in \Lambda_{x}} a|\phi(u)|^{2}\right\}^{q(x)} .
\end{aligned}
$$

The expansion (2.27) bounds $k^{\prime}(W)$ by a product of one-dimensional integrals. We will approximate these by gaussian integrals with mass $m$ and choose $m$ appropriately to give the best bounds. Define

$$
\begin{aligned}
d v_{x}(\phi)= & z^{\prime-1} \prod_{u \in \Lambda_{x}} d \phi(u) \\
& \times \exp \left[-\frac{1}{2} \sum_{b \in \Lambda_{x}^{*}} a|\partial \phi(b)|^{2}-\frac{1}{2} m^{2} \sum_{u \in \Lambda_{x}} a|\phi(u)|^{2}\right],
\end{aligned}
$$

where as usual $z^{\prime}$ normalizes the measure. Then the contribution to (2.27) from a site $x$ (assumed to be neither $z$ nor $w$ ) is

$$
\begin{aligned}
& D^{-1} \int d v_{x}(\phi)\left(\sum_{u \in \Lambda_{x}} a|\phi(u)|^{2}\right)^{q(x)} \\
& \quad \times \exp \left[\left(\sigma+\frac{1}{2} m^{2}+p(x)-2 d\right) \sum_{u \in \Lambda_{x}} a|\phi(u)|^{2}-\lambda \sum_{u \in \Lambda_{x}} a\left(|\phi(u)|^{2}\right)^{2}\right],
\end{aligned}
$$

where the denominator is

$$
D=\int d v_{x}(\phi) \exp \left[\left(\sigma+\frac{1}{2} m^{2}-2 d\right) \sum_{u \in \Lambda_{x}} a|\phi(u)|^{2}-\lambda \sum_{u \in \Lambda_{x}} a\left(|\phi(u)|^{2}\right)^{2}\right] .
$$

At this point we can make simple estimates. The exponent in (2.29) is bounded by

$$
\exp \left[\frac{\beta}{4 \lambda}\left(\sigma+\frac{1}{2} m^{2}+p(x)-2 d\right)^{2}\right]
$$


where we used $\sum_{u \in \Lambda_{x}} a=\beta$, and we will assume that $m^{2}>4 d$. Furthermore the diagonal part of the covariance of (2.28) for real $\phi$ is given by

$$
\int d v_{x}(\phi)|\phi(w)|^{2}=\frac{N}{\beta} \sum_{\substack{k \in Z \\|k| \leqq \frac{\beta}{2 a}}}\left(4 a^{-2} \sin ^{2} \frac{\pi a k}{\beta}+m^{2}\right)^{-1} .
$$

When $\phi$ is complex, the factor $N$ is replaced by $2 N$. This is easily shown to satisfy the bound

$$
\int d v_{x}(\phi)|\phi(w)|^{2} \leqq c_{1} \frac{N}{\beta}\left(\frac{1}{m^{2}}+\frac{\beta}{m}\right),
$$

where $c_{1}$ is a numerical constant. Higher moments may be bounded similarly, in particular

$$
\int d v_{x}(\phi)\left|\phi\left(u_{1}\right)\right|^{2} \ldots\left|\phi\left(u_{p}\right)\right|^{2} \leqq(2 p-1) ! !\left[c_{1} \frac{N}{\beta}\left(\frac{1}{m^{2}}+\frac{\beta}{m}\right)\right]^{p} .
$$

Therefore the numerator in (2.29) is bounded by

$$
\begin{aligned}
\exp & {\left[\frac{\beta}{4 \lambda}\left(\sigma+\frac{1}{2} m^{2}\right)^{2}\right] \int d v_{x}(\phi)\left(\sum_{u \in \Lambda_{x}} a|\phi(u)|^{2}\right)^{q(x)} } \\
& \leqq \exp \left[\frac{\beta}{4 \lambda}\left(\sigma+\frac{1}{2} m^{2}\right)^{2}\right]\left[N\left(\frac{1}{m^{2}}+\frac{\beta}{m}\right)\right]^{q(x)} .
\end{aligned}
$$

Using Jenson's inequality (2.30) is bounded from below by

$$
D \geqq \exp \left[\left(\sigma+\frac{1}{2} m^{2}-2 d\right) \beta \int d v_{x}(\phi)|\phi(u)|^{2}-\lambda \beta \int d v_{x}(\phi)\left(|\phi(u)|^{2}\right)^{2}\right] .
$$

Of course $(2.31) \geqq \frac{N}{\beta m^{2}}$, and so we finally obtain the estimate

$$
\begin{aligned}
(2.29) & \leqq c_{2}\left(\frac{N}{m^{2}}+\frac{N \beta}{m}\right)^{q(x)} \\
& \times \exp \left[\frac{\beta}{4 \lambda}\left(\sigma+\frac{1}{2} m^{2}\right)^{2}-\frac{2 N}{m^{2}}\left(\sigma+\frac{1}{2} m^{2}-2 d\right)+12 \lambda \beta\left(\frac{N}{\beta m^{2}}+\frac{N}{m}\right)^{2}\right] .
\end{aligned}
$$

Until now the value of $m^{2}$ has been arbitrary. We will now choose $m^{2}$ in order to get the best bound.

Lemma 2.4. Let the parameters $\lambda, \sigma, \beta, m^{2}$ satisfy the following conditions;
(i) $m^{2}=\sqrt{\frac{\lambda}{\beta}}$,
(ii) $\beta \sigma^{2} \leqq \lambda$,
(iii) $\beta^{3} \lambda \leqq 1$. 
Furthermore, given any $\varepsilon>0$, let $\beta, \lambda$ satisfy the bound

$$
\beta / \lambda \leqq \varepsilon^{2} .
$$

Then

$$
(2.29) \leqq c_{3}(N \varepsilon)^{q(x)} \exp \left[c_{4} N \varepsilon^{2}+c_{5} N^{2}\right],
$$

where $c_{3}, c_{4}$, and $c_{5}$ are numerical constants.

Lemma 2.4 follows by substituting the given constraints in (2.35).

Returning now to (2.27), we can complete the proof of Proposition 2.2. If $x$ is either $z$ or $w$, the bound (2.36) is multiplied by an additional factor $(N / \sqrt{\beta \lambda})$ coming from the terms $|\phi(z)|^{2},|\phi(w)|^{2}$. The only sites $x \in \Omega$ for which $p(x)$ and $q(x)$ are nonzero are the endpoints of bonds in $W$, and these are less than $2|W|$ of these. Therefore inserting (2.36) into (2.27) gives

$$
\left|k^{\prime}(W)\right| \leqq \frac{N}{\sqrt{\beta \lambda}} \sum_{\{q(x)\}}\left\{c_{3} \exp \left[c_{4} N \varepsilon^{2}+c_{5} N^{2}\right]\right\}^{2|W|}(N \varepsilon)^{|W|},
$$

where we used (2.26). The number of terms in the sum over $\{q(x)\}$ is bounded by $(2 d)^{2|W|}$, and therefore by taking $\varepsilon$ sufficiently small we obtain $(2.20)$ with $M^{\prime}(T) \sim \ln \varepsilon^{-1} \sim \ln (T \lambda)$ as $T \rightarrow \infty$.

Proof of Proposition 2.3. Since $V(b)$ is non-negative for all field values, we have $\exp V(b) \geqq 1$. Combined with the positivity of the measure in the functional integral, this immediately implies the result (2.21).

\section{Absence of High Temperature Symmetry Breaking - The General Case}

The results obtained in the previous section extend in a very general way to an arbitrary model containing gauge fields and scalar matter fields. As before, we define the model on the lattice $\Lambda$ introduced in Sect. II, with periodic boundary conditions in the time direction, and any choice of boundary conditions in the spatial directions. The gauge group $G$ is arbitrary. The scalar field $\phi(x)$ may belong to an arbitrary $N$ dimensional real or complex unitary representation of $G$. If $g$ is an element of $G$, we will write $U(g)$ for the matrix representation of $g$ which acts on the field $\phi$.

An element $g_{b} \in G$ is introduced on each bond $b \in \Lambda^{*}$. The covariant derivative of the scalar field on $b=\langle x, y\rangle$ is then

$$
(D \phi)(b)=\phi(y)-U\left(g_{b}\right) \phi(x)
$$

for a spacelike bond. For a timelike bond there is a factor $a^{-1}$ in the right-hand side of (3.1). We will write $S_{G}$ for the part of the action containing only the gauge field. Our proof works for any real choice of the action $S_{G}$, including of course the Wilson action. The presence of gauge-fixing terms involving only the gauge field is also irrelevant.

The full action for our lattice theory is now given by

$$
S(g, \phi)=S_{G}+\frac{1}{2} \sum_{b \in \Lambda^{*}} a|D \phi(b)|^{2}-\sigma \sum_{x \in A} a|\phi(x)|^{2}+\lambda \sum_{x \in \Lambda} a\left(|\phi(x)|^{2}\right)^{2} .
$$


The corresponding measure which defines the expectation of observables is

$$
Z^{\prime-1} \prod_{b \in \Lambda^{*}} d g_{b} \prod_{x \in \Lambda} d \phi(x) \exp [-S(g, \phi)] .
$$

In order to explore symmetry breaking we again consider two spacelike separated sites $z$ and $w$. Let $P(z, w)$ be any functional of the gauge field $g$, and consider the following observable:

$$
\bar{\phi}(x) \cdot P(z, w) \phi(w) \text {. }
$$

For example, $P(z, w)$ can be the path ordered exponential of gauge fields along a path connecting $z$ and $w$. Then we have the following result concerning the high temperature behavior of this observable.

Theorem 3.1. For any choice of gauge group $G$, of parameters $\lambda, \sigma, a, N$ and of dimension $d \geqq 1$, there is a temperature $0<T^{*}<\infty$ and a function $M(T)$, both depending only on $\lambda, \sigma, N, d$, such that for all $T>T^{*}$

$$
\left.|\langle\bar{\phi}(z) \cdot P(z, w) \phi(w)\rangle| \leqq c N \sqrt{\frac{T}{\lambda}} \exp |-M(T)| z-w \mid\right]\|P(z, w)\|,
$$

where $M(T) \rightarrow \infty$ as $T \rightarrow \infty$, and $c$ is a numerical constant.

Proof of Theorem 3.1. The basic strategy will be to prove convergence of the high temperature expansion for the matter fields in the presence of an arbitrary fixed gauge field. If $\langle\cdot\rangle$ denotes an expectation in the matter field measure

$$
Z_{g}^{-1} \prod_{x \in A} d \phi(x) \exp [-S(g, \phi)]
$$

(with $Z_{g}$ defined to normalize the measure), then the expectation in the complete Higgs theory is given by an average over the background field expectations

$$
\langle\cdot\rangle=Z^{-1} \int \prod_{b \in \Lambda^{*}} d g_{b} Z_{g}\langle\cdot\rangle_{g} \cdot
$$

Consequently, any bound which holds for a background field expectation, uniformly in the gauge field, immediately extends to the complete expectation. Therefore, we will prove:

Lemma 3.2. Under the conditions of Theorem 3.1, and for all gauge fields $g$,

$$
\langle\bar{\phi}(x) \cdot P(z, w) \phi(w)\rangle_{g} \leqq N \sqrt{\frac{T}{\lambda}} \exp [-M(T)|z-w|]\|P(z, w)\| .
$$

This immediately implies Theorem 3.1.

Proof of Lemma 3.2. The argument precisely parallels the treatment in Sect. II. Introducing our cluster expansion yields

$$
\langle\bar{\phi}(z) \cdot \phi(w)\rangle_{g}=\sum_{\substack{W \subset \Omega^{*} \\ z, w \in W \\ \text { connected }}} k_{g}^{\prime}(W) \frac{Z_{g}\left(\Omega^{*} \backslash \bar{W}\right)}{Z_{g}},
$$

where the quantities $k_{g}^{\prime}(W)$ and $Z_{g}(X)$ are the obvious analogues of (2.15) and (2.18). Therefore the proof reduces to the demonstration of the following propositions. 
Proposition 3.3. Let $W \subset \Omega^{*}$ be a connected set containing $z$ and $w$. Then for any choice of parameters $\lambda, \sigma, a, N$, for any dimension $d \geqq 1$ and for any background gauge field $g$, there is a temperature $0<T_{1}<\infty$ and a function $M^{\prime}(T)$, both depending only on $\lambda, \sigma, N, d$ such that for all $T>T_{1}^{*}$

$$
\left|k_{g}^{\prime}(W)\right| \leqq c^{\prime} N \sqrt{\frac{T}{\lambda}} \exp \left[-M^{\prime}(T)|W|\right],
$$

where $M^{\prime}(T) \rightarrow \infty$ as $T \rightarrow \infty$.

Proposition 3.4. For any subset $W \subset \Omega^{*}$,

$$
\frac{Z_{g}\left(\Omega^{*} \backslash \bar{W}\right)}{Z_{g}} \leqq 1
$$

Proof of Proposition 3.3. The proof is almost identical to that given for Proposition 2.2. The only change is in the evaluation of the diagonal part of the one-dimensional covariance of the scalar field. This is formally given by the inverse of the operator $D^{\dagger} D+m^{2}$, where $D$ is the one dimensional covariant derivative with periodic boundary conditions. This depends only on the time component of the gauge field along a timelike loop. By gauge covariance, this gauge field may be replaced by a constant field $g_{0}$ on each bond. In a complex representation $U\left(g_{0}\right)$ can be diagonalised, and the diagonal elements can be written as $e^{i \psi_{1}}, \ldots, e^{i \psi_{N}}$. Then the extension of (2.31) is

$$
\int d v_{x, g}(\phi)|\phi(u)|^{2}=\frac{2}{\beta} \sum_{i=1}^{N} \sum_{\substack{k \in Z \\|k| \leqq \frac{\beta}{2 a}}}\left\{4 a^{-2} \sin ^{2}\left(\frac{\pi a k}{\beta}+\frac{\psi_{i}}{2}\right)+m^{2}\right\}^{-1} .
$$

Since (3.12) is bounded above by its value when $\phi_{i}=0$ for all $i$, the bounds (2.32) and (2.33) still hold. Also (3.12) $\geqq \frac{2 N}{\beta m^{2}}$ is still true, so the proof proceeds exactly as
for Proposition 2.2.

In a real representation, $U\left(g_{0}\right)$ can be brought to a block diagonal form, where each block is either 1 or a $2 \times 2$ rotation matrix. If these rotation angles are $\theta_{1}, \ldots, \theta_{M}, M \leqq \frac{N}{2}$, then the extension of (2.31) is

$$
\begin{aligned}
\int d v_{x, g}(\phi)|\phi(u)|^{2}= & \frac{2}{\beta} \sum_{i=1}^{M} \sum_{\substack{k \in Z \\
|k| \leqq \frac{\beta}{2 a}}}\left\{4 a^{-2} \sin ^{2}\left(\frac{\pi a k}{\beta}+\frac{\theta_{i}}{2}\right)+m^{2}\right\}^{-1} \\
& +\frac{1}{\beta} \sum_{j=2 m+1}^{N} \sum_{\substack{k \in Z \\
|k| \leqq \frac{\beta}{2 a}}}\left\{4 a^{-2} \sin ^{2}\left(\frac{\pi a k}{\beta}\right)+m^{2}\right\}^{-1},
\end{aligned}
$$

and the proof proceeds as before.

Proof of Proposition 3.4. As in Proposition 2.3, our choice of interaction V(b) makes this result trivial. The inclusion of gauge fields changes (2.8) to

$$
V_{g}(b)=\frac{1}{2} \sum_{\langle u, v\rangle \in \Lambda_{b}^{*}} a\left|\phi(u)+U\left(g_{b}\right) \phi(v)\right|^{2} .
$$

Once again, $V_{g}(b)$ is non-negative and this immediately implies the result. 


\section{Symmetry Restoration in the Abelian Higgs Model}

As discussed before, the only lattice gauge theory for which spontaneous symmetry breaking is known to occur is the non-compact abelian Higgs model. In two or more spatial dimensions the global $U(1)$ symmetry of this model is broken at zero temperature, if there is a double-well potential for the scalar field [7]. In this section we will prove that at sufficiently high temperature the global $U(1)$ symmetry is restored.

Because the $U(1)$ gauge field is represented by a non-compact vector field in our theory, some care must be taken in the definition of the finite-temperature functional integral. In addition to the usual gauge-fixing problem, there is a zeromode for the vector field which must be removed.

We will choose boundary conditions so that the lattice $\Lambda$ is periodic in the time direction, with length $\beta$. In the spatial directions however, we will use free boundary conditions, defined by setting the gauge field to zero on all bonds outside the lattice. This removes the zero modes in the spatial directions.

The vector field is represented by a real-valued variable $A(b)$ on each bond $b$ in $\Lambda$. The action for the vector field is a sum of three terms. The first is the usual gauge invariant term $\left(\sum_{b \in p} A(b)\right)^{2}$ for each plaquette $p$ in $\Lambda$, where the sum is over the oriented bonds in $p$. The second is a gauge-fixing term, which we take as $\left(\sum_{b \ni x} A(b)\right)^{2}$ for each $x \in \Lambda$, where the sum is over oriented bonds in $\Lambda$ connected to $x$. Finally to remove the zero mode corresponding to constant timelike vector fields, we introduce the gauge invariant term $\varepsilon\left(\sum_{b_{t}} A\left(b_{t}\right)\right)^{2}$, where the sum is over all timelike bonds $b_{t}$. All our results will be uniform in $\varepsilon$, and we take the limit $\varepsilon \rightarrow 0$ at the end. So the finite-temperature, finite-volume measure for the vector field is

$(D A)=\prod_{b \in \Lambda^{*}} d A(b) \exp \left[-\frac{1}{2} \sum_{p}\left(\sum_{b \in p} A(b)\right)^{2}-\frac{1}{2} \sum_{x}\left(\sum_{b \ni x} A(b)\right)^{2}-\frac{\varepsilon}{2}\left(\sum_{b_{t}} A\left(b_{t}\right)\right)^{2}\right]$.

The scalar field $\phi(x)$ is a one component complex field, which is introduced at each site $x$ in $\Lambda$ as in Sect. II. The only modification to the action (2.1) is the replacement of $\partial \phi$ by the covariant derivative given below. For a spacelike bond $b=\langle x, y\rangle$,

$$
D \phi(b)=\phi(y)-\exp [i e A(b)] \phi(x),
$$

where $e$ is the electric charge. For a timelike bond $b^{\prime}=\left\langle x^{\prime}, y^{\prime}\right\rangle$,

$$
D \phi\left(b^{\prime}\right)=a^{-1}\left(\phi\left(y^{\prime}\right)-\exp \left[i e a A\left(b^{\prime}\right)\right] \phi\left(x^{\prime}\right)\right) .
$$

We will denote by $S_{A}(\phi)$ the action (2.1) with $\partial \phi(b)$ replaced everywhere by $D \phi(b)$, and with the stated boundary conditions. Then the expectation of an observable $F(\phi, A)$ is defined as

$$
\langle F\rangle=\lim _{\varepsilon \rightarrow 0} Z^{\prime-1} \int(D A) \prod_{x \in A} d \phi(x) \exp \left[-S_{A}(\phi)\right] F(\phi, A),
$$

where again $Z^{\prime}$ normalizes the functional integral. The expectation of any observable which is orthogonal to the zero mode in the time direction will be wellbehaved in the limit $\varepsilon \rightarrow 0$. 
In order to investigate symmetry breaking in this model, a "smeared string" observable was introduced in [7]. This observable was locally gauge invariant but was sensitive to breaking of the global $U(1)$ symmetry. We recall the definition below.

Let $z, w$ be spacelike separated sites in $\Lambda$, and denote by $g$ the lattice function which is +1 at $z$ and -1 at $w$, and zero elsewhere. The lattice Laplacian on $A$ is invertible on functions orthogonal to constants, and we denote its inverse on this space by $C$. Then we define the vector field $h$ on each bond $b$ in $A$ by

$$
h(b)=(\partial C g)(b),
$$

where the finite difference derivative is defined in (2.2), (2.3). The observable we use is

$$
K(z, w)=\bar{\phi}(z) \exp \left[i e \sum_{b} A(b) h(b)\right] \phi(w) .
$$

Then in the limit where the spatial lattice is infinite, we define again

$$
M^{2}=\lim _{|z-w| \rightarrow \infty} \lim _{\beta \rightarrow \infty}\langle K(z, w)\rangle .
$$

In [7] it was proved that for $\sigma$ sufficiently large and for $e$ (the electric charge) sufficiently small, and for temporal lattice spacing $a=1$,

$$
M^{2}>0 \text { for } d \geqq 2 \text {. }
$$

The proof in [7] may be easily extended to non-zero temperatures in $d \geqq 3$ by taking $a=1$ and $N_{t}$ any positive integer [12].

This result implies that the global $U(1)$ symmetry is broken at low temperature for the range of parameters indicated. By decreasing the temporal lattice spacing $a$ and holding $N_{t}$ fixed, we can raise the temperature continuously. The following theorem implies that during this process we will inevitably encounter a finite temperature phase transition where the global symmetry is restored.

Theorem 4.1. For any choice of parameters $\lambda, \sigma, a$, e and any dimension $d \geqq 1$, there is a temperature $T^{*}<\infty$ and a function $M(T)$, both depending only on $\lambda, \sigma, d$, such that for all $T>T^{*}$

$$
\langle K(z, w)\rangle \leqq c \sqrt{\frac{T}{\lambda}} \exp [-M(T)|z-w|],
$$

where $M(T) \rightarrow \infty$ as $T \rightarrow \infty$, and $c$ is a numerical constant.

Proof. As in Sect. 3, we define

$$
\langle\bar{\phi}(z) \phi(w)\rangle_{A}=Z_{A}^{-1} \int \prod_{x \in A} d \phi(x) \exp \left[-S_{A}(\phi)\right] \bar{\phi}(z) \phi(w),
$$

where $Z_{A}$ normalizes the expectation. By Lemma 3.2 we know that (4.7) satisfies the required bound (4.6). Since this is uniform in the background field $A$ and in $\varepsilon$, we get the same bound for the full expectation, which is given by

$$
\langle K(z, w)\rangle=\lim _{\varepsilon \rightarrow 0} Z^{\prime-1} \int(D A) Z_{A} \exp \left[i e \sum_{b} A(b) h(b)\right]\langle\bar{\phi}(z) \phi(w)\rangle_{A} .
$$




\section{References}

1. Banks, T., Rabinovici, E.: Finite-temperature behavior of the lattice abelian Higgs model. Nucl. Phys. B160, 349-379 (1979)

2. Borgs, C., Seiler, E.: Lattice Yang-Mills theory at non-zero temperature and the confinement problem. Commun. Math. Phys. 91, 329-380 (1983)

3. Dolan, L., Jackiw, R.: Symmetry behavior at finite temperature. Phys. Rev. D9, No. 12, 3320-3341 (1974)

4. Fradkin, E., Shenker, S.: Phase diagrams of lattice gauge theories with Higgs fields. Phys. Rev. D19, 12, 3682-3697 (1979)

5. Fröhlich, J., Israel, R., Lieb, E., Simon, B.: Phase transitions and reflection positivity. I. General theory and long range lattice models. Commun. Math. Phys. 62, 1-34 (1978)

6. Glimm, J., Jaffe, A.: Quantum physics. A functional integral point of view. Berlin, Heidelberg, New York: Springer 1981, and refs. therein

7. Kennedy, T., King, C.: Spontaneous symmetry breakdown in the abelian Higgs model. Commun. Math. Phys. 104, 327-348 (1986)

8. Ore, O.: Graphs and their uses. Random House New Mathematical Library 1963

9. Osterwalder, K., Seiler, E.: Gauge field theories on a lattice. Ann. Phys. 110, 440-471 (1978)

10. Tomboulis, E., Yaffe, L.: Finite temperature $S U(2)$ lattice gauge theory. Commun. Math. Phys. 100, 313-341 (1985)

11. Weinberg, S.: Gauge and global symmetries at high temperature. Phys. Rev. D9, 3357-3377 (1974)

12. King, C.: Unpublished (1986)

Communicated by A. Jaffe

Received June 4, 1986; in revised form September 10, 1986 